\title{
Erratum to: CAT C-262T and GPX1 Pro198Leu polymorphisms in a Turkish population
}

H. Sinan Suzen • Emel Gucyener • Ozgul Sakalli •

Zuhal Uckun · Gulcin Kose • Duygu Ustel •

Yalcin Duydu

Published online: 8 April 2010

(C) Springer Science+Business Media B.V. 2010

Erratum to: Mol Biol Rep

DOI 10.1007/S11033-009-9540-4

Page 89, Line 5 should be read as sense $5^{\prime}$-TGT GCC CCT ACG CAG GTA CA-3' instead of sense $5^{\prime}$-TGT GCC CCT ACG GTA CA-3'.

The online version of the original article can be found under doi: 10.1007/s11033-009-9540-4.

H. S. Suzen $(\varangle) \cdot$ E. Gucyener · O. Sakalli · Z. Uckun · G. Kose · D. Ustel · Y. Duydu

Department of Toxicology, Faculty of Pharmacy, Ankara

University, Tandogan 06100, Ankara, Turkey

e-mail: suzen@pharmacy.ankara.edu.tr 